\title{
First Magnetic Resonance Imaging-Guided Aortic Stenting and Cava Filter Placement Using a Polyetheretherketone-Based Magnetic Resonance Imaging-Compatible Guidewire in Swine: Proof of Concept
}

\author{
Sebastian Kos • Rolf Huegli • Eugen Hofmann • Harald H. Quick • \\ Hilmar Kuehl · Stephanie Aker • Gernot M. Kaiser • \\ Paul J. A. Borm · Augustinus L. Jacob · Deniz Bilecen
}

Received: 2 October 2008/Accepted: 18 November 2008/Published online: 30 December 2008

(C) Springer Science+Business Media, LLC 2008

\begin{abstract}
The purpose of this study was to demonstrate feasibility of percutaneous transluminal aortic stenting and cava filter placement under magnetic resonance imaging (MRI) guidance exclusively using a polyetheretherketone (PEEK)-based MRI-compatible guidewire. Percutaneous transluminal aortic stenting and cava filter placement were performed in 3 domestic swine. Procedures were performed under MRI-guidance in an open-bore 1.5-T scanner. The applied 0.035 -inch guidewire has a PEEK core reinforced by
\end{abstract}

S. Kos $(\bowtie) \cdot$ A. L. Jacob · D. Bilecen

Institute of Radiology, University Hospital Basel,

Petersgraben 4, 4031 Basel, Switzerland

e-mail: skos@gmx.de

R. Huegli

Institute of Radiology, Kantonsspital Bruderholz,

4101 Bruderholz, Switzerland

E. Hofmann

Vascular Intervention, Biotronik, Ackerstrasse 6,

8180 Buelach, Switzerland

H. H. Quick · H. Kuehl

Department of Diagnostic and Interventional Radiology and Neuroradiology, University of Duisburg-Essen,

Hufelandstrasse 55, 45122 Essen, Germany

\section{S. Aker}

Institute of Pathophysiology, University of Duisburg-Essen,

Hufelansdstrasse 55, 45122 Essen, Germany

\section{G. M. Kaiser}

Department of General, Visceral, and Transplantation Surgery,

University of Duisburg-Essen, Hufelandstrasse 55,

45122 Essen, Germany

P. J. A. Borm

MagnaMedics GmbH, Dennewartstrasse 25-27,

52068 Aachen, Germany fibres, floppy tip, hydrophilic coating, and paramagnetic markings for passive visualization. Through an $11 \mathrm{~F}$ sheath, the guidewire was advanced into the abdominal (swine 1) or thoracic aorta (swine 2), and the stents were deployed. The guidewire was advanced into the inferior vena cava (swine 3), and the cava filter was deployed. Postmortem autopsy was performed. Procedural success, guidewire visibility, pushability, and stent support were qualitatively assessed by consensus. Procedure times were documented. Guidewire guidance into the abdominal and thoracic aortas and the inferior vena cava was successful. Stent deployments were successful in the abdominal (swine 1) and thoracic (swine 2) segments of the descending aorta. Cava filter positioning and deployment was successful. Autopsy documented good stent and filter positioning. Guidewire visibility through applied markers was rated acceptable for aortic stenting and good for venous filter placement. Steerability, pushability, and device support were good. The PEEK-based guidewire allows either percutaneous MRI-guided aortic stenting in the thoracic and abdominal segments of the descending aorta and filter placement in the inferior vena cava with acceptable to good device visibility and offers good steerability, pushability, and device support.

Keywords Interventional radiology - MR-intervention Guide wire · PEEK · MR-guided stenting ·

MR-compatible $\cdot$ Cava filter

\section{Introduction}

Magnetic resonance imaging (MRI) provides excellent softtissue contrast and allows image acquisition and threedimensional reconstruction in any orientation. Compared with fluoroscopy, which is currently used for endovascular interventions, it lacks radiation exposure to the patient and staff and 
may be used without nephrotoxic contrast media. MRI's known ability to depict and/or quantify functional parameters (e.g., blood flow, diffusion, perfusion, etc.) may be used to determine the success of an endovascular intervention [1,2].

However, there have only been some reports on MRIguided endovascular intervention in the literature [1-5]. With modern open-bore MR-systems and fast sequences being available as well as MR-compatible stents, angioplasty balloons and non-braided catheters on the market, this has by most authors been attributed to the lack of an MR-compatible guidewire [6].

In some animal studies, taking the risk of rapid in vivo heating and spark yielding into account, standard nitinol guidewires were used. Sometimes the entire procedure was performed without using a guidewire for device delivery [6-10].

A concept of a MRI-compatible guidewire with a PEEK core was published, but until today the in vivo applicability of such passively marked PEEK-based guidewires has not been proven [11].

The purpose of this study was to show the first in vivo use of an MRI-compatible 0.035-inch PEEK-based guidewire for MRI-guided thoracic and abdominal aortic stenting and cava filter placement in swine.

\section{Materials and Methods}

\section{PEEK-Based Guidewire}

A 0.035 -inch, $160 \mathrm{~cm}$-long MRI-compatible guidewire was used (Biotronik Vascular Intervention, Buelach, Switzerland). It has a central PEEK core (0.57-mm diameter) reinforced by fibres. This core compound is tapered (to $0.15 \mathrm{~mm}$ in diameter) at the distal $12 \mathrm{~cm}$, thereby providing an atraumatic tip. The guidewire core is covered along its axis with a polyurethane jacket and a hydrophilic coating.

It provides passive MRI markings composed of paramagnetic alfa- $\mathrm{Fe}_{3} \mathrm{O}_{4}$ nanoparticles (MagnaFy; MagnaMedics GmbH, Aachen, Germany) (Fig. 1). These nanoparticles were applied on the wire in an adhesive coating, forming $0.03-\mathrm{mm}$ thick and $1.5-\mathrm{mm}$ broad markings. For aortic stenting, four distal markings were spaced by $20 \mathrm{~mm}$. Two further markings were separated by $40 \mathrm{~mm}$, enhancing visibility of the distal part of the guidewire shaft. For cava filter placement, 7 markers were attached, with the distal 5 markers being separated by $20 \mathrm{~mm}$.

\section{MR Imaging and MR Suite}

A 1.5-T whole-body open-bore scanner (Espree; Siemens Healthcare, Erlangen, Germany) was used. With a

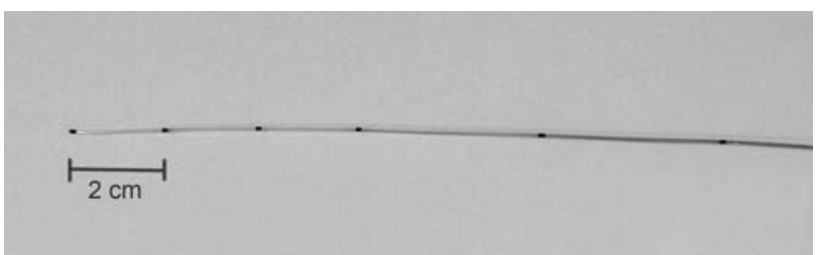

Fig. 1 Photograph of the distal portion of the 0.035-inch PEEKbased MRI-compatible guidewire used in our study. This 0.035-inch guidewire has a soft tip and a hydrophilic coating. For aortic stenting, 6 markers were applied to the wire. The distal 4 passive MRI markings are separated by $20 \mathrm{~mm}$, and 2 markings further proximal are separated by $40 \mathrm{~mm}$

high-performance gradient system (gradient strength $33 \mathrm{mT} /$ $\mathrm{m}$; slew rate $100 \mathrm{~T} / \mathrm{m} / \mathrm{s}$ ) and standard surface receiver coils, a gradient echo T1-FLASH sequence (BEAT-IRT) was used. Imaging parameters were as follows: field of view $400 \times$ $358 \mathrm{~mm}$; matrix size $288 \times 172$; voxel size $2.3 \times 1.4 \times$ $5.0 \mathrm{~mm}$; TR $8.12 \mathrm{~ms}$; TE $5.24 \mathrm{~ms}$; flip angle $21^{\circ}$; bandwidth $299 \mathrm{~Hz} /$ pixel. Images were displayed on an 18-inch monitor (Siemens Healthcare, Erlangen, Germany) mounted beside the MRI-scanner (frame rate $2 / 3$ per second).

Before the intervention, a single intravenous injection of blood-pool contrast media gadofosveset (Vasovist; BayerSchering Pharma, Berlin, Germany) in a dosage of $0.03 \mathrm{mmol}$ gadolinium $(\mathrm{Gd}) / \mathrm{kg}$ body weight (bw) was administered to achieve better vascular depiction throughout the remainder of the procedures.

\section{Animal Preparation}

Animal experiments were conducted at the University Hospital Essen (Germany) according to the policies for the human care and use of animals. The procedures performed were approved by the Regional and Institutional Animal Care and Use Committee.

Three domestic pigs $(60-70 \mathrm{~kg}$ bw) were prepared according to a previously published protocol [12]. Pigs were intubated using an $6.5-\mathrm{mm}$ endotracheal tube and ventilation which was performed throughout the procedure by a respirator (Oxylog; Dräger, Lübeck, Germany). During the entire procedure, infusion anaesthesia was maintained by continuous administration of propofol, midazolam, and fentanyl.

After surgical preparation, $11 \mathrm{~F} / 10-\mathrm{cm}$ catheter sheaths (Radiofocus Introducer II; Terumo, Leuwen, Belgium) were inserted in the iliac arteries and in the iliac vein. A heparin bolus $(5,000 \mathrm{U})$ was given intravenously after sheath placement, and 1,000 $\mathrm{U}$ were given every hour consecutively. Animals were then placed supine and head first into the MRI scanner. 

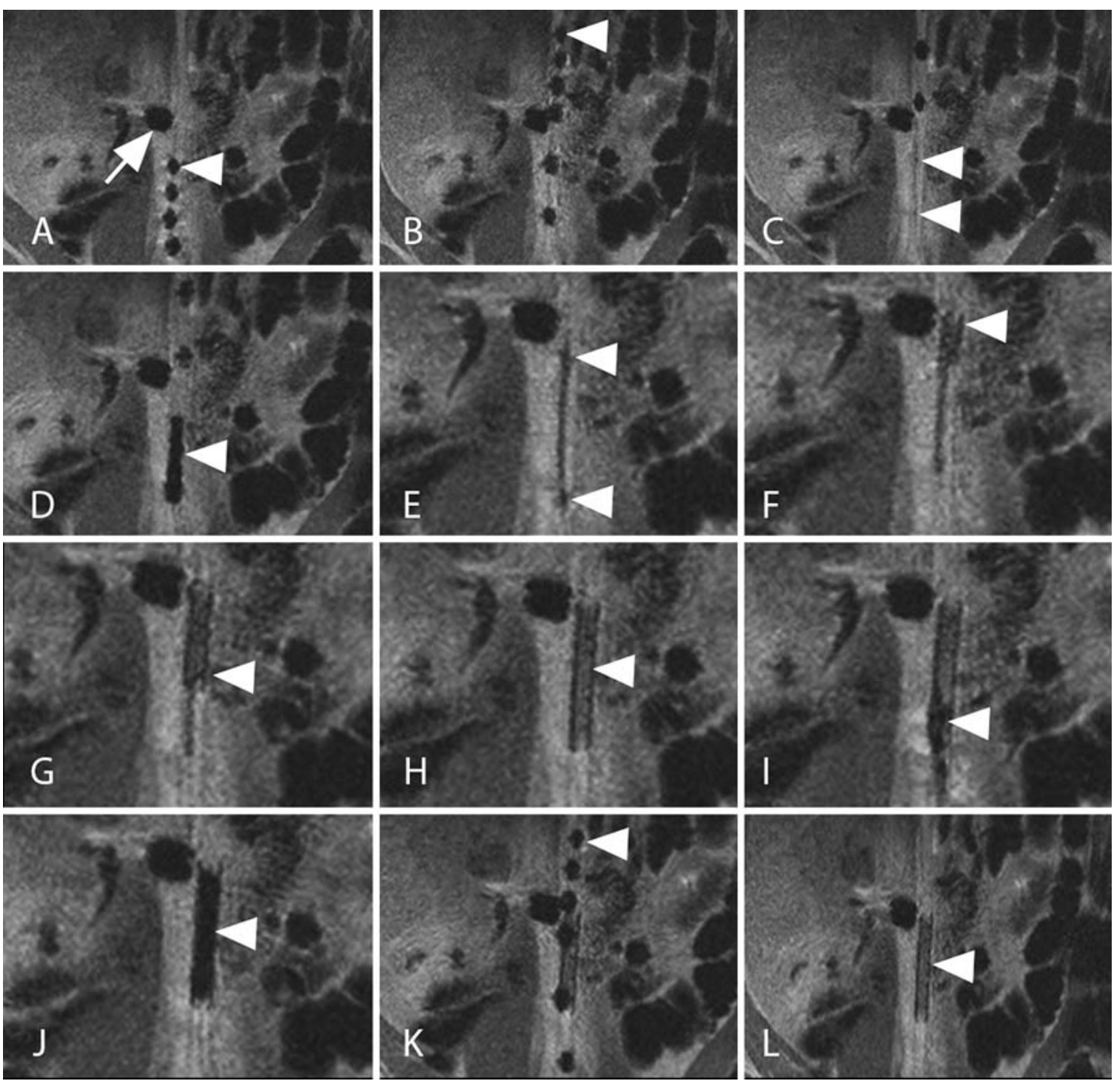

Fig. 2 Abdominal aortic stenting. (A) The right renal artery is marked by a stent implanted earlier (arrow). The guidewire is introduced (A, arrowhead) and advanced into the suprarenal aortic segment (B, arrow). (C) The balloon is inserted over the wire but is poorly visible through slight artefacts caused by the commercially attached standard markers (arrow). (D) The balloon is inflated with

\section{MRI Intervention}

Aortic stent placement and cava filter placement were performed by experienced interventional radiologists in all swine through the iliac catheter sheath and using MRI guidance as previously described.

\section{Abdominal Aortic Stenting: Swine 1}

Through the $11 \mathrm{~F}$ vascular access sheath, the guidewire was advanced into the suprarenal segment of the descending aorta. Over the guidewire, a balloon (Pheron $10 \mathrm{~mm} /$ $40 \mathrm{~mm}$; Biotronik) was placed in the infrarenal abdominal
1-2 ml (0.025 mmol/l) Gd-DOTA (Dotarem) (arrow). (F through $\mathbf{H})$ The balloon is replaced by the self-expandable stent (E, arrowheads), and the stent is sequentially deployed (arrowheads). (J) The balloon is placed in the stent (arrowhead), and dilatation ( $\mathbf{J}$, arrowhead) is performed within the stent. Balloon and guidewire are removed $(\mathbf{K}$, arrowhead), and the stent position is documented (L, arrowhead)

aorta, followed by percutaneous transluminal angioplasty (PTA) through inflation of the balloon with 1-2 ml (0.025 mmol/l) Gd-DOTA (Dotarem; Guerbet, Roissy CDG Cedex, France). Thereafter, a self-expandable stent was positioned in the infrarenal abdominal aorta and deployed (Astron $10 \mathrm{~mm} / 60 \mathrm{~mm}$ ). A second balloon dilatation was performed within the stent (Pheron $10 \mathrm{~mm} /$ $40 \mathrm{~mm}$; Biotronik).

\section{Thoracic Aortic Stenting: Swine 2}

Through the left 11 vascular access sheath, the guidewire was advanced into the thoracic segment of the descending 

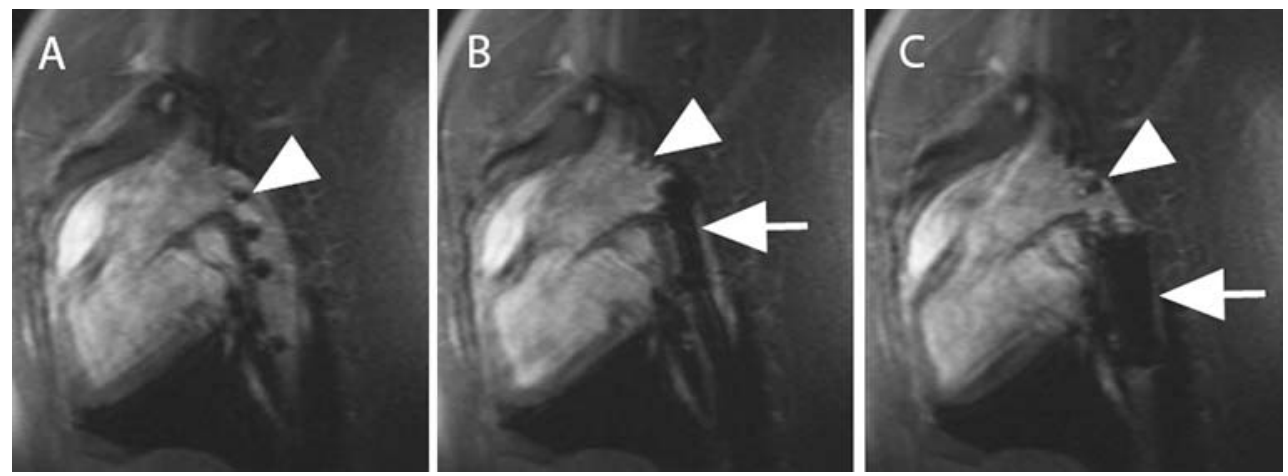

Fig. 3 Thoracic aortic stenting. The guide PEEK-based guidewire (A through $\mathbf{C}$, arrowheads) is advanced into the descending thoracic segment of the aorta $(\mathbf{A})$. The stent (arrow) is advanced over the wire $(\mathbf{B})$, positioned, and released $(\mathbf{C}$, arrow)

Fig. 4 Photographs of autopsy documentation. A Stent in the right renal artery (arrow). The infrarenal abdominal aortic stent (A, arrowhead) and the stent in the descending thoracic aortic segment (B, arrowhead) are marked. An incision in the proximal descending aortic segment exposed the proximal end of the thoracic stent. After preparation of the liver and partial exposition of the inferior vena cava, the inferior vena cava filter can be seen $(\mathbf{C}$, arrow). Longitudinal incision of the ventral wall of the inferior vena cava demonstrates the good positioning and full and correct deployment of the placed cava filter (D, arrow)
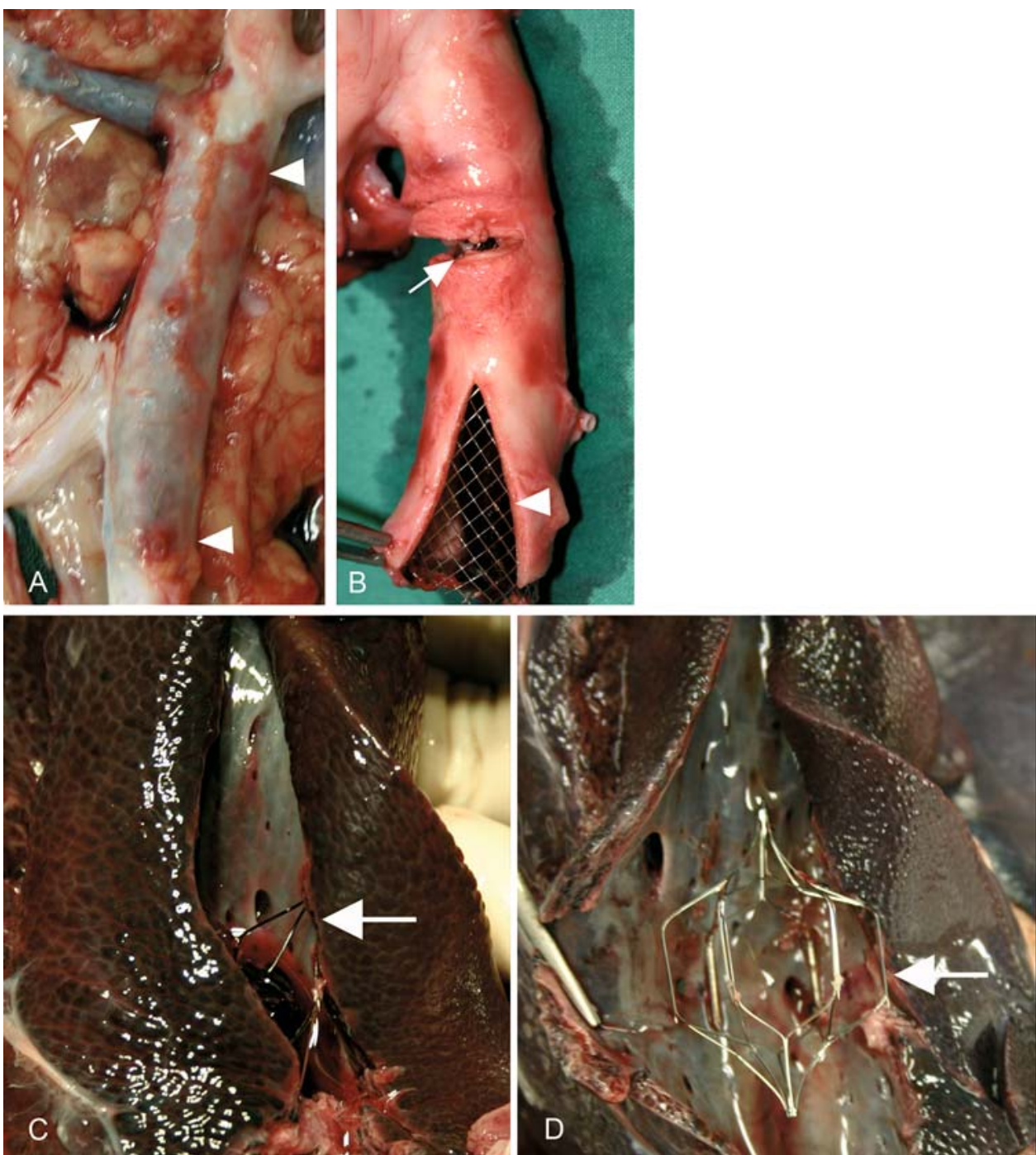

aorta. Over the guidewire, the self-expandable stent (Wallstent $22 \mathrm{~mm} / 45 \mathrm{~mm}$; Boston Scientific, Maple Grove, MN) was positioned in the descending thoracic aorta beyond the origin of the left subclavian artery and released.
Cava Filter Placement: Swine 3

Through the venous iliac $11 \mathrm{~F}$ access sheath, the guidewire was advanced 2 times into the hepatic segment of the inferior vena cava. Over the positioned guidewire, the 


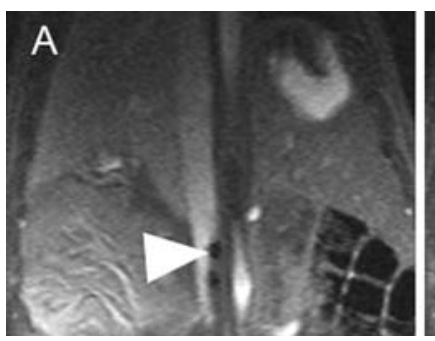

Fig. 5 First phlebographic use of the guidewire (coronal view). (A through C) Under real-time MRI-guidance with FLASH sequence, the wire is sequentially advanced in the inferior vena cava. First, the two most distal markers are depicted (A, arrowhead). The guidewire is

filter's 6F (internal diameter), $90 \mathrm{~cm}$-long introducer sheath OptEase; Retrievable Vena Cava Filter; Cordis Europe, Roden, The Netherlands) was advanced into inferior vena cava. Because of the atypically deep retroperitoneal position of the right kidney, the infrarenal segment of the inferior vena cava was short and did not allow filter placement below the renal veins. The sheath, which was marked only by a regular X-ray-absorbing marker at the tip, was therefore positioned twice in the hepatic segment of the inferior vena cava. Correct intravenous positioning of the sheath was documented by hand injection of a $10-\mathrm{ml}$ bolus $(0.05 \mathrm{mmol} \mathrm{Gd} / \mathrm{ml})$ Gd-DOTA (Dotarem). The guidewire was withdrawn and the selfcentering 6 diamond-shaped cava filter (Optease Retrievable Vena Cava Filter; Cordis Europe, Roden, The Netherlands) positioned and released in the hepatic segment of the inferior vena cava.

Procedure times were documented for the interventions in all swine. Success rates ( 2 grades: \pm ) of any procedure step were rated, and guidewire characteristics — such as visibility, pushability, steerability, and filter/balloon/stent support (3 grades: poor [+]; acceptable $[++]$; and good $[+++]$ - - were assessed by consensus among the interventional radiologists.

After the experiments, the swine were killed by intravenous injection of phenobarbital $(80 \mathrm{mg} / \mathrm{kg})$. The positions of the deployed stents and the cava filter were documented by postmortem autopsy.

\section{Results}

\section{Aortic Stenting}

Guidewire guidance into the targeted abdominal and thoracic aortic segments was successful ( 2 of 2). Over-the-wire placement and dilatation of the PTA balloon in the abdominal aorta was feasible before and after stent deployment ( 2 of 2). Over-the-wire positioning and release of self-expandable stents was successful (2 of 2) (Figs. 2,3). advanced into the hepatic segment of the inferior vena cava so that first the distal 5 markers (B, arrowhead) are visualized, and then all seven markers are in the field of view (C, arrowheads)

Postmortem autopsy showed good stent positioning and no evidence of traumatic vessel wall damage (Fig. 4). Procedure times were 12 minutes for stenting of the thoracic aortic segment and 10 minutes for stenting of the abdominal aortic segment. The guidewire, its surface, and the applied passive marking macroscopically remained intact. There was no evidence of guidewire fracture or kinking. By consensus, guidewire visibility was rated acceptable $(++)$ and guidewire pushability, steerability, and balloon/stent support were rated good $(+++)$.

\section{Cava Filter Placement}

Guidewire positioning in the hepatic segment of the inferior vena cava was successful as was placement of the cava filter's introducer sheath over the wire (Figs. 5, 6). After removal of the guidewire, positioning and release of the filter through the introducer sheath was successful. Postmortem autopsy showed good filter positioning in the hepatic segment of the inferior vena cava (Fig. 4). Autopsy showed no evidence of traumatic vessel wall damage. Procedure times was 6 minutes. The PEEK-based guidewire and its passive markings remained intact, without macroscopic evidence of guidewire fracture or kinking. By consensus, guidewire visibility was rated good $(+++)$ and guidewire pushability and introducer sheath support were rated good $(+++)$.

\section{Discussion}

This results of this study demonstrate that MRI-guided stenting of the aorta and cava filter placement are feasible when using an MRI-compatible PEEK-based guidewire. Commercially available nonbraided catheters, PTA balloons, and stents may safely be used for MRI-guided endovascular interventions as shown earlier in different vascular territories. The first MRI-guided placement of a vena cava filter, which required 30 seconds for the acquisition of a single imaging slice, was described in 1997 [13]. With faster sequences available today, 2 more recent studies 

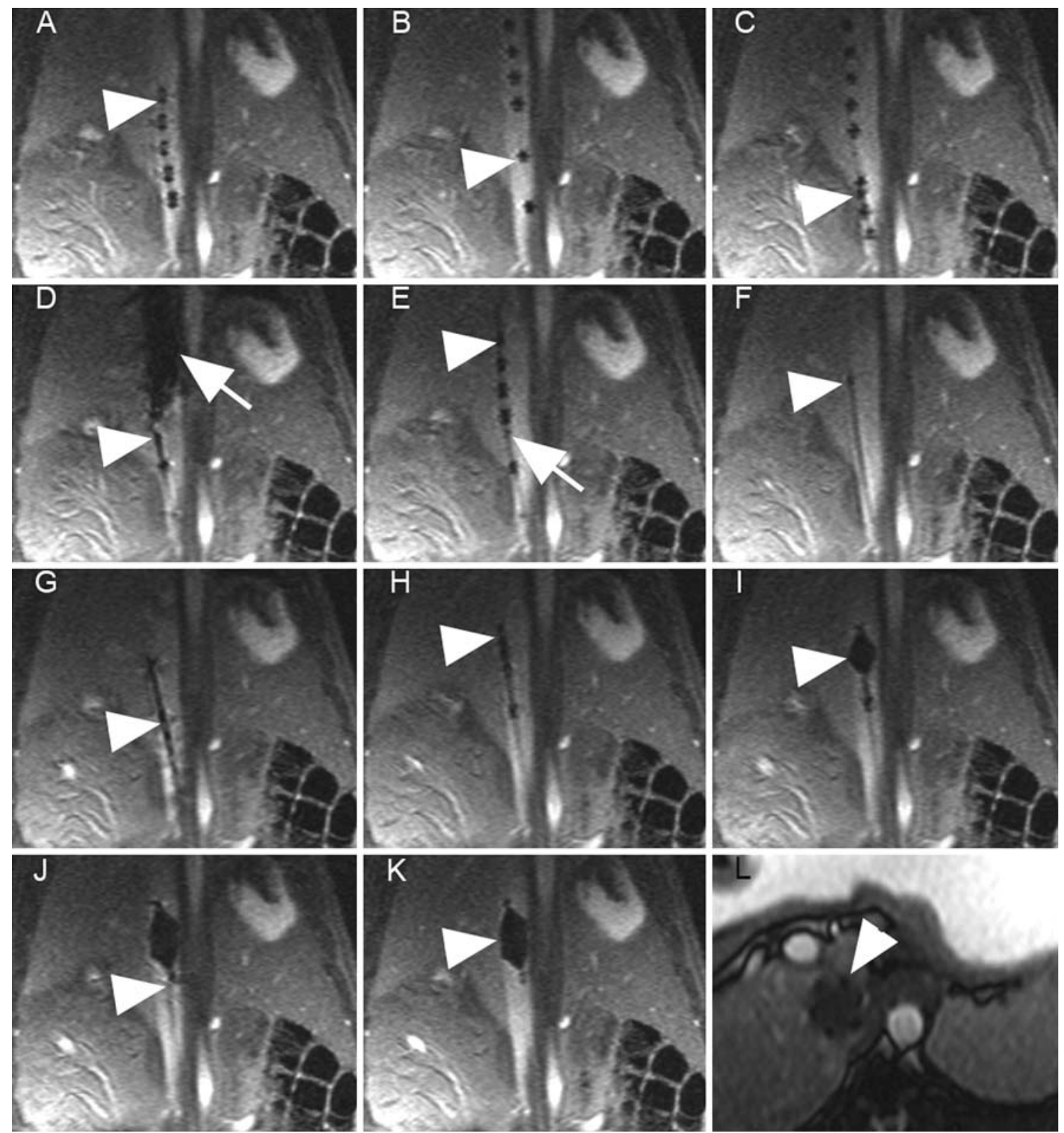

Fig. 6 MR-guided placement of the inferior vena cava filter. (A, B) The guidewire is sequentially advanced into the hepatic segment of the inferior vena cava so that all 7 MRI markings are in the field of view (arrowheads). (C, D) The unmodified 6F filter introducer sheath is advanced over the wire into the hepatic segment of the vein (arrowhead). (E) The standard X-ray-absorbing marker of the sheath (arrow) only causes a weak artefact in the magnetic field. (D) After guidewire removal, correct intravenous sheath positioning is documented applying a contrast media flush through the introducer sheath

showed the feasibility cava filter placement in swine under real-time MRI-guidance [14, 15]. However, given the previously described advantages of MRI for the guidance of endovascular procedures, until today this promising technique has not been shown to be transferable to humans.

This can be explained, as such animal studies did not use a guidewire for procedure guidance, thereby increasing the (arrow). (E) The guidewire is the reinserted (arrowhead) through the introducer sheath $(\mathbf{E}$, arrow). (F) The sheath is advanced more centrally (arrow), and the wire is removed. (G through I) With the sheath in place, the filter is then pushed into and through the sheath (arrowheads). (J) After full deployment of the filter, the introducer sheath (arrowhead) is removed (K). (L) Axial images of the hepatic segment of the inferior vena cava document full deployment of the filter, and 6 straight filter struts are depicted in the periphery of the vein (arrowhead)

risk to cause vessel dissection, perforation or rupture, or as a major drawback, observed sparking and guidewire heating in the magnetic field, when using standard nitinol guidewires. This crucial limitation, potentially harmful for the patient and the operator, may now be overcome by the use of the PEEK-based MR-compatible guidewire. The guidewire's mechanical properties, such as its good device 
support capabilities and pushability, are promising, and the floppy wire tip allows atraumatic procedure guidance. The attached paramagnetic markings provide at least acceptable device visibility in gradient-echo sequences. This passivemarker approach allows simple handling of the guidewire and leads to short procedure time. This is beneficial, compared with other more complicated active-tracking approaches (e.g., guidewire receiver coils) applied earlier [16-20]. The first concepts of automated tracking for passively marked devices have been investigated and may facilitate procedures in the future [21]. Modification of the passive marking's size and thickness may optimize vessellumen contrast and allow marker use according to the diameter of the targeted vessel. The commercially available stents and balloons used in our study were chosen according to target vessel size and product availability.

The OptEase cava filter used in our study is known to be safely usable in the magnetic field [22]. In this study, the filter and its introducer sheath were applied as commercially available without additional MRI markings. This lead to suboptimal visibility of the sheath, through a weak artefact caused by the X-ray-absorbing marker at its tip. For suboptimal visibility of the introducer sheath, sheath positioning was verified in this study through the marker and additional free-hand bolus contrast-media injection through the sheath. Thereby the introducer sheath was safely introduced and precisely positioned over the wire. Because of the atypical deep inflow of the right renal vein in our swine, suprarenal filter placement was performed in the inferior vena cava, which has been shown to be safe and effective in humans [23].

In the future, application of the described passive markings to balloons, stents, filters, introducer sheaths, and catheters (if needed) may facilitate depiction of such over-the-wire devices for MRI-guided endovascular procedures.

Intravenous application of blood-pool contrast media provides good vascular depiction throughout the interventions. Its higher relaxivity and longer blood half-life (average distribution phase 0.48 hours) compared with standard extracellular MRI contrast medias, may allow endovascular procedure control with a single injection of contrast media in the future [23-25].

Acknowledgments This study was designed as a first-proof concept study in swine. Of course, further investigation is necessary to determine the potential of this new PEEK-based guidewire for MRIguided arterial and venous interventions.

\section{References}

1. Kos S, Huegli R, Bongartz G et al (2008) MR-guided endovascular interventions: a comprehensive review on techniques and applications. Eur Radiol 18:645-657
2. Bock M, Wacker F (2008) MR-guided intravascular interventions: techniques and applications. J Magn Reson Imaging 27:326-338

3. Mahnken A, Chalabi K, Jalali F et al (2004) Magnetic resonanceguided placement of aortic stents grafts: feasibility with real-time magnetic resonance fluoroscopy. J Vasc Interv Radiol 15:189-195

4. Omary RA, Frayne R, Unal O et al (2000) MR-guided angioplasty of renal artery stenosis in a pig model: a feasibility study. J Vasc Interv Radiol 11:373-381

5. Bucker A, Neuerburg J, Adam G et al (2003) MR-guided coil embolisation of renal arteries in an animal model. Rofo 175: 271-274

6. Eggebrecht H, Kuhl H, Kaiser GM et al (2006) Feasibility of realtime magnetic resonance-guided stent-graft placement in a swine model of descending aortic dissection. Eur Heart J 27:613-620

7. Nitz WR, Oppelt A, Renz W et al (2001) On the heating of linear conductive structures as guide wires and catheters in interventional MRI. J Magn Reson Imaging 13:105-114

8. Omary R, Gehl J, Schirf B et al (2006) MR imaging versus conventional X-ray fluoroscopy-guided renal angioplasty in swine: prospective randomized comparison. Radiology 238: 489-496

9. Konings MK, Bartels LW, Smits HF et al (2000) Heating around intravascular guidewires by resonating RF waves. J Magn Reson Imaging 12:79-85

10. Buecker A, Spuentrup E, Schmitz-Rode T et al (2004) Use of a nonmetallic guide wire for magnetic resonance-guided coronary artery catheterization. Invest Radiol 39:656-660

11. Mekle R, Hofmann E, Scheffler K et al (2006) A polymer-based MR-compatible guidewire: a study to explore new prospects for interventional peripheral magnetic resonance angiography (ipMRA). J Magn Reson Imaging 23:145-155

12. Kaiser G, Breuckmann F, Aker S et al (2007) Anesthesia for cardiovascular interventions and magnetic resonance imaging in pigs. J Am Assoc Lab Anim Sci 46:30-33

13. Neuerburg J, Bucker A, Adam G et al (1997) Cava filter placement under MRI control. Experimental in vitro and in vivo studies. Rofo 167:418-422

14. Shih MC, Rogers WJ, Hagspiel KD (2006) Real-time magnetic resonance-guided placement of retrievable inferior vena cava filters: comparison with fluoroscopic guidance with use of in vitro and animal models. J Vasc Interv Radiol 17:327-333

15. Bucker A, Neuerburg JM, Adam GB et al (2001) Real-time MR guidance for inferior vena cava filter placement in an animal model. J Vasc Interv Radiol 12:753-756

16. Glowinski A, Adam G, Bucker A et al (1997) Catheter visualization using locally induced, actively controlled field inhomogeneities. Magn Reson Med 38:253-258

17. Dumoulin CL, Souza SP, Darrow RD (1993) Real-time position monitoring of invasive devices using magnetic resonance. Magn Reson Med 29:411-415

18. Quick HH, Kuehl H, Kaiser G et al (2003) Interventional MRA using actively visualized catheters, TrueFISP, and real-time image fusion. Magn Reson Med 49:129-137

19. Quick HH, Zenge MO, Kuehl H et al (2005) Interventional magnetic resonance angiography with no strings attached: wireless active catheter visualization. Magn Reson Med 53:446-455

20. Ocali O, Atalar E (1997) Intravascular magnetic resonance imaging using a loopless catheter antenna. Magn Reson Med 37:112-118

21. Rauschenberg J, de Oliveira A, Muller S et al (2007) An algorithm for passive marker localization in interventional MRI. Z Med Phys 17:180-189

22. Shellock FG (2008) Reference manual for magnetic resonance safety, implants and devices: 2008 edition. Biomedical Research Publishing Group, Los Angeles CA, p 422 
23. Leiner T, Schoenberg S (2007) Current status of renal artery magnetic resonance imaging: theoretical and practical considerations and the potential role of blood-pool contrast agents. Eur Radiol 17(Suppl. 2):B13-B17
24. Meaney J, Goyen M (2007) Recent advances in contrast-enhanced magnetic resonance angiography. Eur Radiol 17(Suppl 2):B2-B6 25. Bremerich J, Bilecen D, Reimer P (2007) MR angiography with blood pool contrast agents. Eur Radiol 17:3017-3024 